\title{
The aesthetic management of gingival enlargement and hyperpigmentation in maxillary anterior region: a case report
}

\author{
Shafira Kurnia*
}

\begin{abstract}
Objective: To describe the aesthetic treatment stage of gingival enlargement with gingival hyperpigmentation. Determination of the type of action detail will assist many clinicians in diagnosis and treat gingival enlargement with hyperpigmentation.

Methods: The 17-year-old male patient, coming with his enlarged gums. Patients claimed to have no hypertension, blood disorders, diabetes and hepatitis. After general check-up and early treatment, gingivectomy

The oral administration administered was $500 \mathrm{mg}$ tid amoxicillin and 500mg tid.prn mefenamic acid.

Results: After gingivectomy has performed multiple diastema appears and suggested orthodontia treatment.

Conclusion: The combination of gingivectomy treatment with gingival depigmentation may be performed simultaneously using a scalpel periodontal instrument.
\end{abstract} gingival depigmentation were performed using scalpel no. $15 \mathrm{c}$.
Department of Periodontology, Faculty of Dental Medicine, Airlangga University, Surabaya, Indonesia

${ }^{*}$ Correspondence to:

shafira-k-s@fkg.unair.ac.id

Received: 6 December 2018

Revised: 20 January 2019

Accepted: 25 March 2019

Keywords: Gingival enlargement, Gingival hyperpigmentation, Gingivectomy, Gingival depigmentation

Cite this Article:Kurnia S. 2019. The aesthetic management of gingival enlargement and hyperpigmentation in maxillary anterior region: a case report. Journal of Case Reports in Dental Medicine. 1(1): 1-4. D0I: 10.20956/jcrdm.v1i1.82

\section{Introduction}

Diseases with the etiology of developmental abnormalities, inflammation or neoplasms may manifest in the oral mucosa. ${ }^{1}$ The gingival enlargement lesion are the most commonly affected mucosa. ${ }^{2}$ This lesion is a manifestation of reaction from tartar irritation, mastication, broken teeth, dentures and excessive teeth restoration. ${ }^{3}$

Gingival enlargement is a common formation that occurs due to inflammation of the gingiva. ${ }^{4}$ The cause is multifactorial that is the interaction between the host and the environment and its response to stimuli. Factors such stimuli include plaques associated with hormonal disorders and manifestations of blood disorders such as leukemia, thrombocitopenia, or trobositopathy. This enlargement can cause mastication, speech, aesthetic and physiological problems. The most common enlargement is due to plaque induced inflammatory reactions. Enlargement may occur locally or spread throughout the teeth. ${ }^{5}$

Hyperpigmentation gingival is a dark purple, brown and light brown gingival discoloration with irregular shapes. Gingival hyperpigmentation is the result of a buildup of melanin granules produced by melanoblasts. Melanin is a brown pigment nonhemoglobin derivate. Melanin is the most common endogenous pigment produced by melanocyte found in the basal cell layer and suprabasal epithelium. ${ }^{6}$

This case report showed reported a gingival enlargement and gingival hyperpigmentation followed by gingival depigmentation action through a periodontal surgical procedure that is gingivectomy performed on the same day.

\section{Case Report}

The 17 years old male patient came with enlarged gums. Patients do not smoke and deny having hypertension, blood disorders, diabetes, hepatitis. He also has a good general condition and good extraoral conditions. Clinically, gingival enlargement and hyperpigmentation of the gingiva especially occur in the maxillary anterior teeth figure 1. Appeared teeth do not come into contact well in the proximal region. At the time of probing bleeding. There is a false pocket of 4 to $4.5 \mathrm{~mm}$ on the maxillary anterior region. Examination of panoramic images indicates a multiple diastema in the maxillary anterior teeth figure 2 .

Clinical examination showed gingiva enlargement 111213212223313233414243 , bleeding on probing and inflammation 1112132122233132 33414243 , calculus and plaque of maxillary and mandibular teeth, malposition 111221223132 41 42. The was diagnosed as Chronic marginal gingivitis 111213212223313233414243 . The etiology observed was dental plaque and overall prognosis was good. Treatment plan including dental health education and scaling root planing as non-surgical therapy. Gingivectomy on maxillary region was planned on surgical phase. Maintenance and observation were suggested every four months 
for regular check.

Initial treatment of scaling root planning and dental health education prior to surgery phase was done. Gingivectomy performed one week after initial treatment and performed if there is no inflammation figure 3A. Gingival contour right after gingivectomy shown in figure $3 \mathrm{~B}$. Amoxicillin 500mg and mefenamic acid 500mg was administered three times daily.

Figure 4 first control five days after surgical phase shown redness in operating area, but no pain was felt by the patient. Patient was instructed to use chlorhexidine oral gargle twice a day for two weeks. Second control two weeks after surgical phase showed good result on the gingival with minimal redness and no pain. After four months, patient received restoration treatment figure 5 .

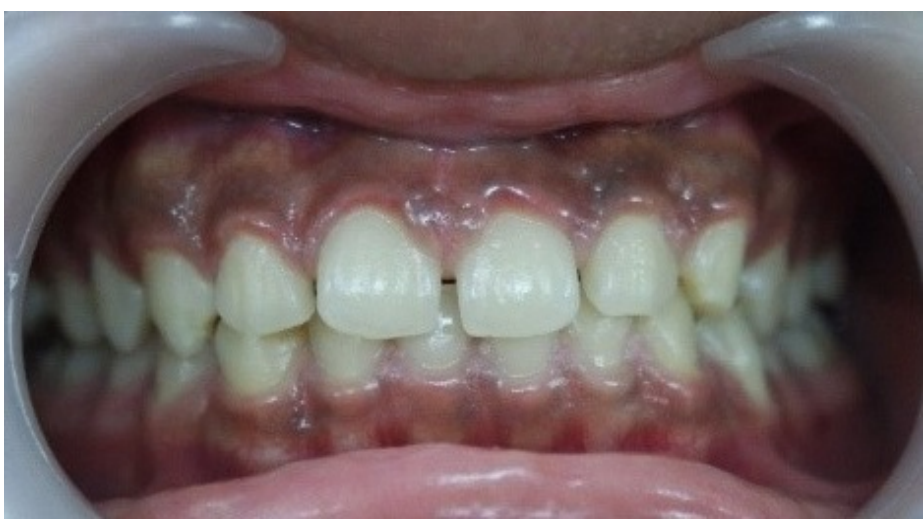

\section{Figure 1 Clinical photos of patients before treatment, gingival enlargement and hyperpigmentation of maxillary anterior teeth}

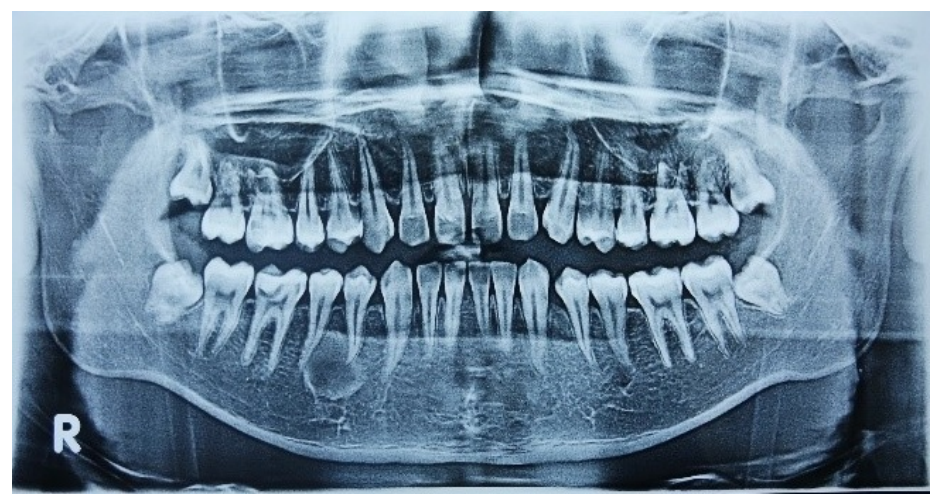

Figure 2 Panoramic photos, there appears to be multiple diastema in the maxillary anterior teeth

\section{Discussion}

The case of gingival enlargement or gingival overgrowth varies greatly. Mostly caused by inflammation due to plaque. Plaque causes a continuous and long-lasting stimulation resulting in chronic irritation resulting in fibrous connective tissue proliferating. ${ }^{7.8}$ Plaques and bacteria that accumulate for a long time lead to infectious infiltration of the cell. This condition can be overcome with conventional initial treatment such as scaling and root planning. ${ }^{9}$ When gingival enlargement remains persistent after conventional treatment then subsequent treatment is periodontal surgical treatment which includes gingivectomy, flap technique using LASER, electrocauter. In this case gingivectomy is performed. . $^{8,9}$

In this case, the clinical prior to treatment appears a blackish-gingival color in the keratinized area. This blackish color is gingival hyperpigmentation caused by the accumulation of melanin granules produced by melanoblasts located between the epithelial cells in the basal layer of the epithelium..$^{10}$ Gingival hyperpigmentation is not a malignancy or a periodontal disease. However, hyperpigmentation conditions can be very disturbing aesthetically. The procedure undertaken to remove gingival hyperpigmentation is depigmentation using a periodontal knife orban tool performed after gingivectomy on the same day. ${ }^{11}$

The abnormal gingival size of enlargement and widening in the interdental and cervical areas can be a reservoir of germs and plaques that can lead to inflammation. Bleeding during probing shows there is inflammation. In this case the shape and size of the teeth is normal but shows poor contact between the teeth. On examination of panoramic images there are multiple diastema in the anterior teeth. Based on anamnesa, this has been going on for a long time even the patient cannot remember when the gingival enlargement occurs. Patients claimed to have no blood disorders, hypertension, diabetes and hepatitis. This condition is not only very disturbing aesthetically but also create a sense of discomfort because every brushing gums always bleed. A complete blood examination was performed to determine the patient's systemic condition. One week after initial treatment is scaling and root planning, patients feel bleeding when brushing teeth. But gingival enlargement is still visible. The next action plan is gingivectomy and gingival depigmentation. At the 5th day of control, the periodontal pack is opened and looks reddish and bleeding in the cervical area of area. 

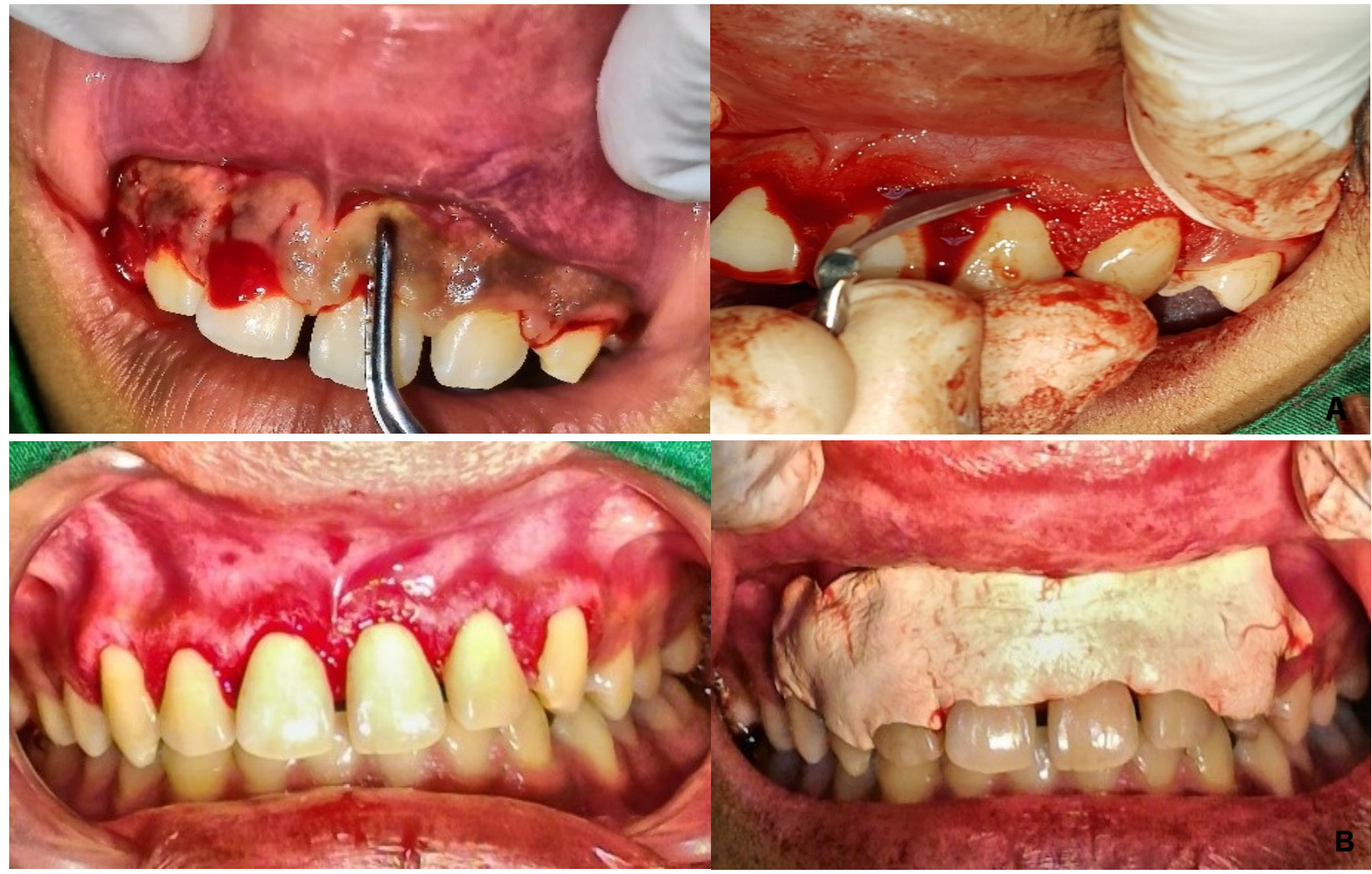

Figure 3 A. Gingivectomy procedure: pocket marked with pocket marker, then inserted $1 \mathrm{~mm}$ with scalpel no .15c in apical bleeding point (left). Gingival depigmentation, using periodontal knife orban, erodes the surface of the epithelial layer (right), B. Results right after gingivectomy and gingival depigmentation (left). Application of periodontal packs in the operating area (right).
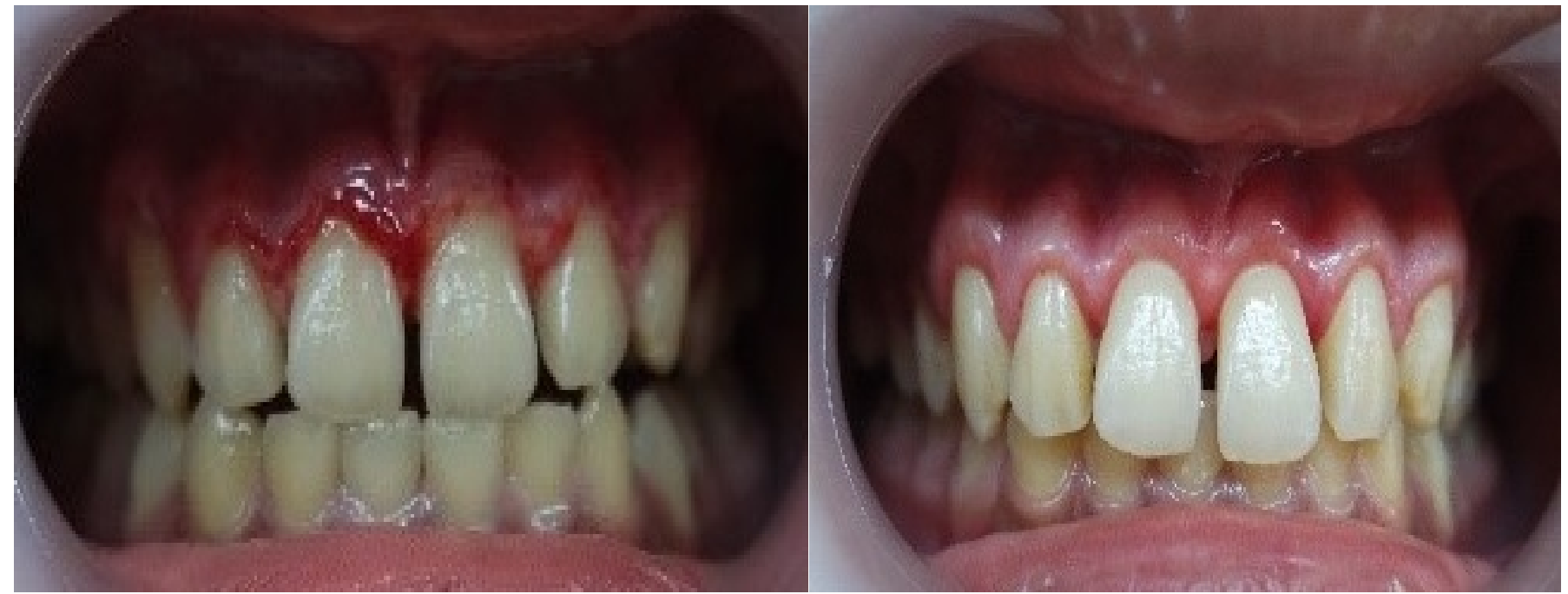

Figure 4 The control condition of the 5 th postoperative day appears to be redness in the cervical area (left). Control condition II day 14 postoperatively, bleeding visible, normal gum color (right). 


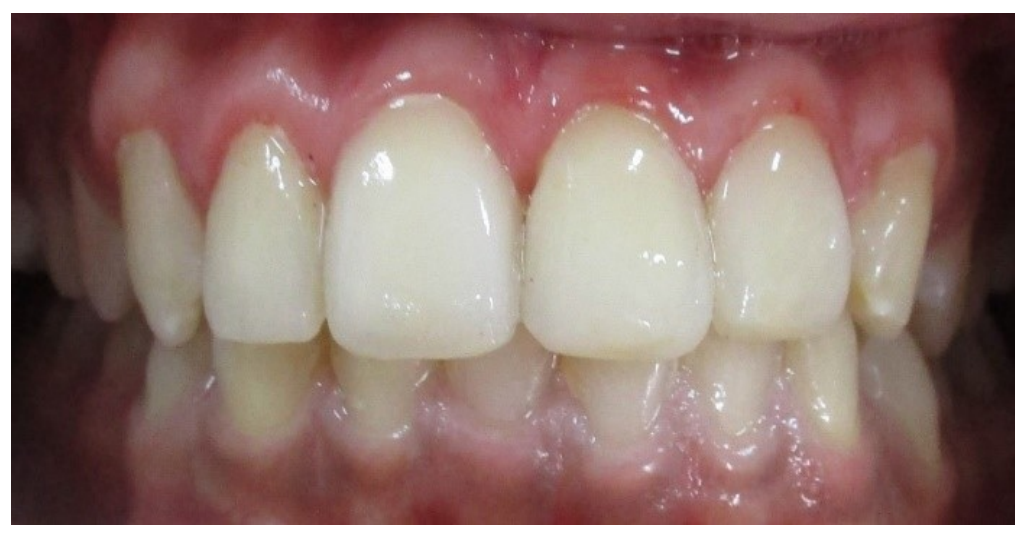

Figure 5 Control four month postoperative, normal gum color, anterior teeth already restored to improve contact point

Suspected irritation of the surgical area due to poor oral hygiene of the patient. Saline irrigation and $\mathrm{H} 2 \mathrm{O} 2$ are performed to reduce irritation and remove debris in the wound area. At 14th day control, the wound seemed to improve, the gingival color returned to normal, the bleeding did not exist. The next plan of action is to send the patient for orthodontic treatment but the patient refuses for some reason. Restoration measures taken to prevent the occurrence of hyperplasia recurrence is to restore the dental crown with direct veneer so it is expected good dental contact. Direct veneer treatment is performed by the dental conservation department.

\section{Conclusion}

Understanding the pathogenesis of gingival enlargement is important. Local factors such as plaque and calculus play an important role in gingival enlargement. Similarly gingival hyperpigmentation, although not a malignancy or periodontal disease, often disrupts esthetics so this disrupt patient's social life. In this case report, the size of the gingival enlargement and gingival hyperpigmentation conditions is a patient's major problem that needs to be addressed immediately. In this case report also explained the importance of periodontal surgery to eliminate gingival enlargement and gingival depigmentation and how to motivate patients to maintain post-treatment oral hygiene.

\section{Aknowledgment}

The authors would like to express sincere gratitude to Britaria Theressy for her help during case treatment.

\section{Conflict of Interest}

The authors report no conflict of interest.

\section{References}

1. Effiom OA, Adeyemo WL, Soyele OO. Focal reactive lesions of the gingival: an analysis of 314 cases at tertiary health institution in Nigeria. Niger Med J 2011;52: 35-40.

2. Nartey NO, Mosadomr HA, Al-Cailani $M$, et al. Localised inflammatory hyperplasia of the oral cavity: clinico pathological study of 164 cases. Saudi Dent J 1994;6: 145-50.

3. Zarei MR, Chamani G, Amanpoor S. Reactive hyperplasia of the oral cavity in Kerman Province, Iran: A review of 172 cases. Br J Oral Maxillofac Surg 2007;45: 288-92.

4. Trackman P, Kantarci A. Connective tissue metabolism and gingival overgrowth. Crit Rev Oral Bio Med 2004;15: 165-175.

5. Carranza FA, Hogan EL. Gingival enlargement. In: Newman MG, Takei HH, Klokkevold PR, Carranza FA. Carranza's Clinical Periodontology. 11th ed. Philadelphia: W.B. Saunders Company; 2006. p. 373-390.

6. Tal H, Oegiesser D, Tal M. Gingival depigmentation by erbium: YAG laser: Clinical observations and patient responses. Journal of Periodontology. 2003;74: 1660-1667.

7. Tiwana PS, Kok IJ, Stoker DS, et al. Facial distortion secondary to idiopathic gingival hyperplasia: surgical management and oral reconstruction with endosseous implants. Oral Surg Oral Med Oral Pathol Oral Radiol Endod. 2005;100: 153-157.

8. Agrawal N, Agrawal K, Mhaske S. An uncommon presenta tion of an inflammatory gingival enlargement responding to non-surgical periodontal therapy. Int J Dent Hyg 2011;9: 303-307.

9. Buddiga V, Ramagoni NK, Mahantesh H. Gingival enlargement - a case series. Ann Essence Dent 2012;1: 73-76.

10. Atsawasuwan P, Greethong K, Nimmanon V. Treatment of gingival hyperpigmentation for esthetic purposes by Nd: YAG laser: report of 4 cases. J Periodonto 2000;71: 315-321.

11. Tjiptoningsih UG. Enlargement gingival treatment on teeth 11 and 21. J Dentomaxillofac Sci 2016;1: 196-200.

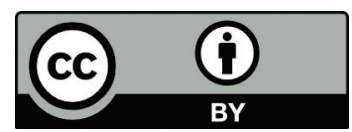

This work is licensed under a Creative Commons Attribution 